\title{
Prediction of Genotypic Values for Apple Fruit Texture Traits in a Breeding Population Derived from 'Honeycrisp'
}

\author{
Steven J. McKay \\ Department of Horticultural Science, University of Minnesota, 305 Alderman Hall, 1970 Folwell \\ Avenue, St. Paul, MN 55108
}

James M. Bradeen

Department of Plant Pathology, University of Minnesota, 495 Borlaug Hall, 1991 Upper Buford

Circle, St. Paul, MN 55108

\author{
James J. Luby ${ }^{1}$ \\ Department of Horticultural Science, University of Minnesota, 305 Alderman Hall, 1970 Folwell \\ Avenue, St. Paul, MN 55108
}

\begin{abstract}
Additional INDEX words. Malus, BLUP, sensory evaluation, crispness, firmness, juiciness
Abstract. The commercially successful apple (Malus pumila Mill.) cultivar Honeycrisp is known for its high degrees of crispness and juiciness. This cultivar has been incorporated into numerous breeding programs in an effort to duplicate its desirable texture traits in conjunction with such other traits as reduced postharvest disorders, disease resistance, and improved tree vigor. This study characterizes variability and estimates heritability for several apple fruit texture traits within a large breeding population over several years. Five full-sib families, all sharing 'Honeycrisp' as a common parent, were assayed with respect to crispness, firmness, and juiciness using sensory evaluation panels and total work required to fracture tissue using instrumental methods. The incomplete block design of the sensory panels, coupled with best linear unbiased prediction, facilitated the evaluation of a large number of genotypes with small numbers of fruit per genotype while accounting for individual sensory panelist effects. Broad-sense heritability estimates exceeded 0.70 for all four traits. Principal component analysis, applied to the phenotypic data, characterized 'Honeycrisp' as having average crispness and low firmness (53rd percentile relative to its offspring) but also as being a relatively extreme example of high juiciness and low work to fracture (first percentile). The improved characterization of desired fruit texture phenotypes and the high levels of broad-sense heritability provide valuable tools for the further development of new, high-quality apple cultivars.
\end{abstract}

Domesticated apple [Malus pumila (synonymous with Malus $\times$ domestica Borkh.)] is among the most commercially important temperate tree fruit in the world with nearly 72 million tonnes produced globally in 2009 (Food and Agricultural Organization of the United Nations, 2010). A large number of globally important apple cultivars (e.g., Delicious, Golden Delicious, Granny Smith) are of unknown parentage or the products of open-pollinated seed, often dating to the 19th century. In contrast, a small number of more recent, successful introductions (e.g., 'Fuji', 'Gala', 'Cripps Pink', 'Honeycrisp') are the results of breeding programs. Numerous biological features in apple hinder the rapid development of new cultivars, including self-incompatibility, high heterozygosity, extended juvenility, and long generation times (e.g., Janick et al., 1996). The polygenic nature of many fruit quality traits serves as an additional complicating factor in fruit breeding programs.

Fruit texture traits such as crispness, firmness, juiciness, and mealiness are cited as a primary component of consumer choice

Received for publication 30 June 2011. Accepted for publication 14 Sept. 2011. We thank Ken Mullen for assistance in the field and with preparing apple samples for sensory evaluation panels. Cindy Tong, Andy David, and three anonymous reviewers provided very useful feedback.

This article represents a portion of the thesis submitted in partial fulfillment of the Doctor of Philosophy degree of the first author.

${ }^{1}$ Corresponding author. E-mail: 1ubyx001@umn.edu. when purchasing fresh fruit (Péneau et al., 2006), so improved texture characteristics that increase fruit marketability are among the goals in apple breeding programs worldwide (e.g., Janick et al., 1996). Sensory evaluations are frequently used in conjunction with instrumental studies as a result of the complex nature of fruit texture traits and the importance of consumer perception of these traits (e.g., Chauvin et al., 2010; Karlsen et al., 1999). Sensory panels have been used to dissect the texture trait characteristics of apple genotypes, frequently a small number of named cultivars (e.g., Daillant-Spinnler et al., 1996; Harker et al., 2002; Karlsen et al., 1999). Sensory panels are also incorporated in later stages of fruit breeding programs to estimate such genetic parameters as heritability, combining ability, and variance components (e.g., Alspach and Oraguzie, 2002; Oraguzie et al., 2001) or to assess consumer preferences among advanced selections (Hampson et al., 2000). Typically all panelists evaluate all genotypes, restricting the number of samples that can be evaluated in a single study. Furthermore, this method requires numerous samples for each genotype, a limiting condition in the first several years of fruit production, especially in the early stages of a breeding program when genotypes are represented by single trees.

The commercially successful cultivar Honeycrisp, introduced by the University of Minnesota in 1991, is known for its high degrees of crispness and juiciness as well as for its long storage capacity (e.g., Allan-Wojtas et al., 2003; Mann et al., 2005). This cultivar has been incorporated into numerous breeding programs 
in an effort to duplicate its desirable texture traits in conjunction with such other traits as improved disease resistance and tree vigor. Several thousand seedlings, exhibiting a wide range of texture traits, have been created at the University of Minnesota by crossing 'Honeycrisp' with numerous other cultivars. This study endeavors to characterize variation in a large breeding population with respect to several apple fruit texture traits using instrumental and sensory methods and to estimate heritability for each of the traits.

\section{Materials and Methods}

Plant material. Five full-sib families from crosses made between 1992 and 1998, with 'Honeycrisp' as a common parent, were selected from among the breeding population at the University of Minnesota's Horticultural Research Center in

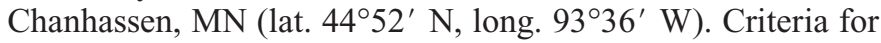
inclusion were number of fruit-bearing genotypes, variability in texture traits among genotypes (observed in previous years), reciprocal crosses within families, when possible, and no known common ancestry other than through the 'Honeycrisp' parent (Table 1).

The other parents included two unnamed advanced selections from the University of Minnesota breeding program (MN1702 and MN-1764), two named North American cultivars (Jonafree and Monark), and PI279323, an accession of the English cultivar Pitmaston Pineapple (hereafter referred to as Pitmaston). Reciprocal crosses were available for the first three families. Numerous additional genotypes, representing several parents, purchased fruit, and other genotypes, were phenotyped simultaneously with the rest of the cultivars. Data from these non-progeny genotypes were retained in the analyses to assist in the prediction of panelist effects but are not discussed further.

HARVEST, SAMPLE PREPARATION, AND RANDOMIZATION. Apples were harvested weekly in 2005, 2006, and 2007 starting in August. To harvest fruit at similar stages of maturity, the starch-iodine test (Blanpied and Silsby, 1992) was selected as an easily performed and replicable proxy assessment of maturity rather than as an explicit measure of edibility. Genotypes whose fruit consistently scored 5 to 7 on the Blanpied and Silsby scale on a given day were designated as mature and a minimum of four fruit per tree was collected. All harvested fruit was stored in labeled paper bags and refrigerated at $4{ }^{\circ} \mathrm{C}$ within 1

Table 1. Distribution of genotypes across apple families (indicated by non-'Honeycrisp' parent) and parental role of 'Honeycrisp'.

\begin{tabular}{lcccl}
\hline $\begin{array}{l}\text { Non-'Honeycrisp' } \\
\text { parent }\end{array}$ & $\begin{array}{l}\text { 'Honeycrisp' } \\
\text { as maternal } \\
\text { parent (no.) }\end{array}$ & $\begin{array}{c}\text { 'Honeycrisp' } \\
\text { as paternal } \\
\text { parent (no.) }\end{array}$ & $\begin{array}{c}\text { Total } \\
\text { (no.) }\end{array}$ & $\begin{array}{c}\text { Distinguishing trait } \\
\text { of non-'Honeycrisp' } \\
\text { parent }\end{array}$ \\
\hline MN-1702 & 30 & 22 & 52 & $\begin{array}{c}\text { Firm and long-storing } \\
\text { MN-1764 }\end{array}$ \\
Jonafree & 3 & 116 & 119 & $\begin{array}{c}\text { Crisp texture, softens rapidly in } \\
\text { storage }\end{array}$ \\
Monark & 13 & 41 & 54 & $\begin{array}{l}\text { Scab-resistant } \\
\text { Pitmaston }\end{array}$ \\
Non-progeny & 54 & 0 & 84 & Fruit mature early \\
& 54 & 0 & 54 & Distinctive flavor and russetting \\
& NA & NA & 152 & \\
\hline
\end{tabular}

${ }^{\mathrm{z}}$ Non-progeny trees were phenotyped simultaneously and included in statistical analyses to improve the prediction of panelist effects.

'Not applicable, because the parental role of 'Honeycrisp' was unknown or 'Honeycrisp' was not a parent. per genotype per year. h of completing the daily harvest. Fruit were harvested only once

Within 1 week of harvest, four apples per tree were evaluated by sensory panels with respect to sensory traits. Panelists were recruited from students, staff, and affiliates of the University of Minnesota Department of Horticultural Science and the Applied Plant Sciences graduate program. Panelists were untrained other than by written directions concerning how to hold, bite, and chew the samples and at what point to make judgments. Panels were held once per week with a single exception during which 2 weeks' worth of apples were evaluated simultaneously. The employment of multiple sensory panels, reflecting the multiple harvests, reduced variability resulting from postharvest physiological effects by allowing for uniform storage time before evaluation (e.g., Mann et al., 2005). The repeated employment of panelists throughout the duration of the study, as opposed to different panelists each week, reduced the confounding of panelist effects with week-of-harvest effects.

A single quadrant was removed from the same region of each apple, midway between the sun-exposed and shaded portions of each fruit, cut parallel to the central axis of the core, and the central core tissue was removed. These quadrants were then halved, again parallel to the central axis of the core, producing two $45^{\circ}$ wedges per fruit, which were presented to panelists as a single sample. The remaining three-quadrant portions of the apples were returned to refrigeration at $4{ }^{\circ} \mathrm{C}$ for additional assays. Samples were presented peel side down to panelists in white paper baking cups arrayed in three rows and four columns on white foam supermarket food trays. Because panelist effects were to be directly estimated, control genotypes were not included in the samples. Randomization schemes were constructed on the same days as the panels, allowing for panelists to confirm their availability on each evaluation date. Fruit samples were randomized according to incomplete randomized block designs with panelists designated as blocks. In 2005, weekly cyclic designs were constructed (John and Williams, 1995); whereas in 2006 and 2007, weekly Latinized $\alpha$ designs were constructed using CycDesigN 2.1 (John and Williams, 1995; Whitaker et al., 2004). In this manner, a large number of trees could be evaluated without requiring that all genotypes be evaluated by all panelists. Sample order varied across panelists to reduce systematic bias as a result of panelist fatigue. Genotype identity was retained for each apple section but not revealed to panelists. Additionally, genotypes were frequently replicated within panelists to address consistency of panelists within genotypes (two apples from the same tree). Four fruit per genotype were evaluated by the sensory panel, except as just noted.

Sensory evaluation. Sensory panels assessed three sensory traits for each apple: crispness, firmness, and juiciness. Following written instructions, panelists first evaluated crispness after biting each apple wedge once with the incisors and then evaluated firmness and juiciness by chewing each section five to 10 times with the molars (Allan-Wojtas et al., 2003; Harker et al., 2003). Panelists recorded their assessments, 
making vertical marks along a 16-cm generalized labeled magnitude scale (Fig. 1; Green et al., 1993). Locations of rating marks were later measured and divided by $16 \mathrm{~cm}$ to derive values between 0.00 and 1.00 .

Texture analyzer. After the sensory panels, the sun-exposed regions of the remaining three-quadrant apple portions were assayed with a texture analyzer (TA.XT2i; Stable Micro Systems, Godalming, U.K.). A 6-mm-diameter cylindrical plug was removed from each apple parallel to the fruit's central core, and a triple-beam snapping test was performed with a blunt blade (e.g., Mann et al., 2005). The blade was applied at a constant velocity of $0.8 \mathrm{~mm} \cdot \mathrm{s}^{-1}$ until the apple samples fractured. The texture analyzer recorded the force [gram-force $(\mathrm{gf})$ ] required to maintain this constant velocity, and the distance (millimeters) traversed at the sampling frequency of 200 data points per second. Total work, from the moment of blade contact with the sample until the moment of fracture, was calculated for each time step using the formula: $1 \mathrm{~J}=1 \mathrm{~N} \cdot \mathrm{m}^{-1}=1.01972 \times 10^{5} \mathrm{gf} \cdot \mathrm{mm}^{-1}$. The cumulative sum yielded an integrated measure of total work, reflecting total energy expended and not assuming constant force throughout the probe's motion.

Although most samples were assayed within $1 \mathrm{~d}$ of the sensory panels, delays of up to 1 week or more occasionally occurred for logistical reasons. To assess the impact of storing non-intact fruit, additional intact fruit from the same genotypes were frequently included in the texture analyzer samples. These fruit allowed for an investigation into the change in total work of non-intact fruit relative to the intact fruit with the objective of determining whether intact and non-intact fruit were interchangeable, at least over small time periods. All fruit, intact and non-intact, were stored at $4{ }^{\circ} \mathrm{C}$ until instrumental assay.

Statistical analyses. Linear mixed-effects models were constructed for each of the three sensory traits and the total work variable. Family and direction of cross within family were estimated as fixed effects, yielding best linear unbiased effects; whereas genotype, year, and panelists were incorporated as random effects as were interaction terms, yielding best linear unbiased predictions [BLUP (e.g., Piepho et al., 2008)]. Before analysis, the data were re-parameterized to reflect the unbalanced nesting of genotypes within families and directions of reciprocal crosses (Piepho et al., 2006). For example, binary variables were created to represent each family. Three additional binary variables were created to indicate the reciprocal crosses for each of the three affected (MN-1702, MN-1764, and 'Jonafree') families. Finally, five variables were created to indicate the genotypes contained within each of the five families. Thus, direction of cross and genotype variables were nested only within their appropriate families, eliminating extraneous overparameterization. The three sensory trait values were logittransformed before analysis to constrain predicted values within

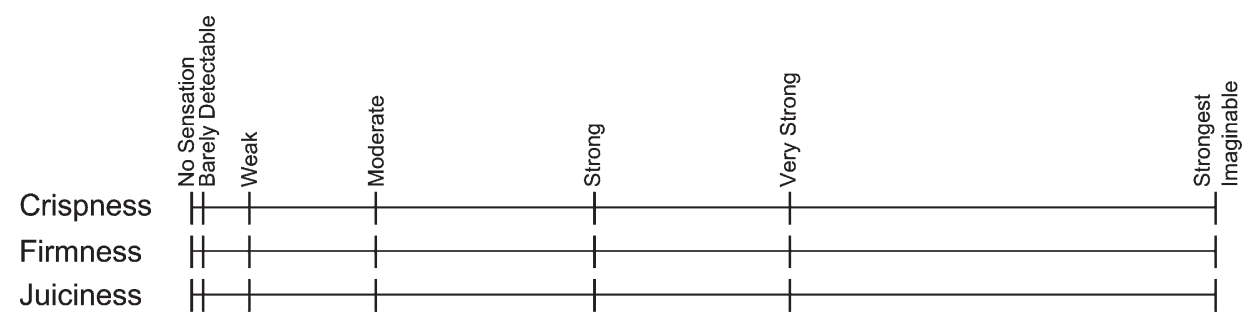

Fig. 1. Labeled magnitude scale used by apple sensory evaluation panels (modified from Green et al., 1993). the interval $[0.00,1.00]$. All statistical analyses were performed using R 2.6.1 (R Development Core Team, 2007).

For each trait, a full, or saturated, model was constructed, and a backward-stepwise regression method was applied to sequentially remove statistically nonsignificant terms. The initial full model for each sensory trait was

$$
\operatorname{logit}(\mathrm{y})=\alpha / \beta / \gamma+\delta+\eta+((\alpha / \beta / \gamma) \times \delta)+(\eta \times \delta)+\varepsilon
$$

The first term, $\alpha / \beta / \gamma$, represents family $(\alpha)$, direction of cross nested within family $(\beta)$, and genotype nested within direction of cross $(\gamma)$, respectively. The next two terms, $\delta$ and $\eta$, represent year and panelist effects, respectively. The last three terms, $((\alpha / \beta / \gamma) \times \delta),(\eta \times \delta)$, and $\varepsilon$, model the genetic-by-year interactions with nesting as before, panelist-by-year interaction, and the residual term. Family and direction of cross within family were modeled as fixed effects, whereas all other terms were modeled as random effects. The nested parameterization of the various genetic components allows for nonconstant variance across family/direction of cross combinations. Including the panelists as a random term reflects their role as blocking structures.

To evaluate the contributions of fixed effects, preliminary models were first constructed treating all variables as fixed, and models were compared by means of analyses of variance. Subsequent decisions regarding model selection with respect to random effects were based on likelihood ratio tests [constructed through restricted maximum likelihood (ReML)] and the Akaike information criterion; both methods weigh the goodness of fit of the models as well as their complexity (Pinheiro and Bates, 2002). Significance levels were established at $P<0.05$.

The initial model for the total work variable was equivalent with a few modifications. First, the work variable was not transformed with the logit function. Second, all panelist terms were omitted, because these data were collected independently of the sensory panels. Third, a new term, $\mu$, nested within the interaction term, was added to reflect whether a sample came from an intact fruit or from a fruit that had been sampled for the sensory panels. The full model was $\mathrm{y}=\alpha / \beta / \gamma+\delta+((\alpha)$ $\beta / \gamma) \times \delta) / \mu+\varepsilon$.

An additional linear mixed-effect model was constructed for each of the three sensory traits to assess the consistency of panelists' evaluations of multiple fruit per genotype. Data were restricted to those data corresponding to multiple fruit per genotypes evaluated by a single panelist. Panelist and genotype within panelist were incorporated into the model to assess the variability across panelists' assessments of genotypes relative to variability within genotypes. The logit-transformed variables were modeled according to the equation $\operatorname{logit}(\mathrm{y})=\eta / \gamma+\varepsilon$. All terms were modeled as random effects.

Heritability terms were estimated using a bootstrap method (Piepho and Möhring, 2007). First, the final fitted model for each trait was used to simulate a data set by means of the "simulate" function contained within the lme4 package for R (Bates, 2007), incorporating the variance-covariance structure of the fitted linear model and reflecting the unbalanced nature of the original data set. Each simulated 
data set was, in turn, fitted according to the same linear model. The fitted values were retained and the correlation value was calculated for the simulated data set and its corresponding fitted values. This procedure was repeated 1000 times for each trait, yielding 1000 correlation values. The mean of these simulated correlations is asymptotically equivalent to the broad-sense heritability, $\mathrm{H}^{2}$ (Piepho and Möhring, 2007). The 95th percentile intervals for each estimate was calculated as the interval between the 2.5th percentile and the 97.5 th percentile.

Before back-transformation with the inverse logit transformation, panelist-adjusted sensory values were calculated by subtracting model-derived predictions of panelist and panelistby-year terms from the fitted values of each apple. Correlation values were calculated among the panelist-adjusted values for the three sensory traits and predicted values for the instrumental trait.

In light of the collinearity of the four texture traits, a principal component analysis (PCA) was performed to further characterize the texture traits within the population. Panelist-adjusted sensory trait values and predicted instrumental values were averaged across years before analysis. To compare 'Honeycrisp' and the other two parental genotypes included in this study (MN-1702 and MN-1764) to the five families, principal component scores for the three parents were estimated using the loadings derived from the PCA; parental data were standardized using the same means and scalar multipliers as were used in the PCA.

\section{Results}

Data Collection. Over 3 years, a total of 515 genotypes were sampled, 391 of which were contained within the five families (Table 1). Weekly harvest sample sizes ranged from eight to 102 genotypes with an average of 42.0. A total of 23 panelists participated over the course of the study, five for a single year, nine for 2 years, and nine for all 3 years. Week-to-week variation in panelist availability yielded average annual panel sizes of 13.8, 13.0, and 10.6 for the 3 years, respectively. Variation in both weekly sample sizes and weekly panel sizes resulted in an average of 15.3 fruit per panelist per week with sample sizes exceeding two dozen in only 2 of the 23 weeks.

Prediction of Genotypic values. Family effects and direction of cross within family were both statistically significant for crispness $(P<0.001)$. 'Honeycrisp' as the male parent resulted in a $15 \%$ increase in crispness when crossed with MN1702 but $17 \%$ and $14 \%$ decreases when crossed with MN-1764 and 'Jonafree', respectively. Year did not contribute significantly to the variability $(P=1.000)$ nor did the interactions of year with family or direction of cross within family $(P=0.949$ and $P=0.933$, respectively). Interactions of genotype and panelist with year both contributed significantly $(P<0.001$ for both) to the variability of the data. In contrast, neither firmness nor juiciness exhibited significant contributions from the direction of cross within family terms $(P=0.071$ and $P=0.245$, respectively). Otherwise, the final models for both traits were the same as for crispness.

Neither family nor direction of cross within family were statistically significant for the instrumental trait of total work to fracture $(P=1.000$ and $P=0.233$, respectively). Familyby-year and tree-by-year interaction terms were statistically significant $(P<0.001$ for both). Unlike the three sensory traits, year of harvest contributed significantly $(P<0.001)$ to the variability of this instrumental trait.
Panelists' evaluations of multiple apples from within a single tree were very consistent relative to evaluations of apples from multiple trees on the same date. For each of the three sensory traits, the variability among genotypes was $\approx 60 \%$ greater than the variability within genotypes with respect to both crispness and firmness and essentially equal with respect to juiciness. In other words, panelists' evaluations largely reflected the expected distribution of variability among genotypes exceeding variability within genotypes.

The large sample sizes allowed for the identification of statistically significant correlations among the sensory and instrumental traits at the genotype level (Table 2). Instrumental work values between intact fruit and non-intact fruit, averaged within genotype-by-year, were highly correlated $(r=0.75, P<$ 0.001).

The first two principal components (PC1 and PC2) accounted for $44 \%$ and $28 \%$ of the total variability displayed among the mean annual values (Table 3 ). PC1 captures the collinearity of the four texture traits, whereas the remaining principal components reflect deviations from this collinearity. PC2 reflects a high juice-low work to low juice-high work spectrum.

The inclusion of three of the parental genotypes in this study's population provides a means of comparing their predicted phenotypic values with those of the five families (Table 4). The predicted phenotypic values of three parental cultivars reflect, in part, the rationale behind their inclusion in the University of Minnesota apple breeding program. 'Honeycrisp' expressed average crispness (55th percentile) and high juiciness levels (95th percentile) but low firmness and very low total work to fracture (31st and 15 th percentiles, respectively) relative to other genotypes. This combination of texture values is expressed as a moderate value with respect to PC1 (53rd percentile) and an extreme value with respect to PC2 (first percentile; Fig. 2). In contrast, MN-1702, expressing higher levels of crispness and firmness, much higher levels of total

Table 2. Correlations between panelist-adjusted fruit texture trait best linear unbiased prediction (BLUP) values for all five apple families averaged across years. ${ }^{\mathrm{z}}$

\begin{tabular}{lccc}
\hline & Crispness & Firmness & Juiciness \\
\hline Firmness & $0.85^{\mathrm{y}}$ & & \\
Juiciness & $0.60^{\mathrm{y}}$ & $0.41^{\mathrm{y}}$ & \\
Total work to fracture & $0.47^{\mathrm{y}}$ & $0.62^{\mathrm{y}}$ & 0.05 \\
\hline
\end{tabular}

${ }^{\mathrm{z}}$ Sensory trait (crispness, firmness, and juiciness) values are panelistadjusted. Total work to fracture is measured on the same (non-intact) fruit as the sensory traits. Sensory traits are logit-transformed. ${ }^{\mathrm{y}}$ Correlation values statistically significant at the $P<0.001$ level.

Table 3. Principal component (PC) loadings, and corresponding percentages of total variability explained, derived from fruit texture trait best linear unbiased prediction (BLUP) values, averaged across years, of all five apple families.

\begin{tabular}{lcrrr}
\hline & PC1 & PC2 & PC3 & PC4 \\
\hline Crispness & -0.58 & -0.16 & -0.37 & -0.70 \\
Firmness & -0.58 & 0.17 & -0.43 & -0.67 \\
Juiciness & -0.37 & -0.75 & 0.51 & -0.21 \\
Total work to fracture & -0.43 & 0.62 & 0.64 & 0.12 \\
Percentage of total & $44 \%$ & $28 \%$ & $17 \%$ & $11 \%$ \\
$\quad$ & & & & \\
\hline
\end{tabular}


work to fracture, and reduced juiciness relative to 'Honeycrisp', displays more moderate values with respect to the first two principal components (30th and 60th percentiles, respectively). MN-1764 seems most exceptional for low levels of crispness, firmness, and total work to fracture, and its 90th percentile ranking with respect to $\mathrm{PC} 1$ reflects these low levels (the negative loadings for this component indicate that a high percentile corresponds with low trait values). The second percentile ranking of the second principal component, however, captures the juiciness for which this tree was originally included in the breeding program. Restricting comparisons of parental values to individual families yields similar results (data not shown).

Heritability estimation. Despite the identification of numerous sources of variability during the model fitting process, the bootstrap analysis estimate yielded broad-sense heritability terms in excess of 0.7 for all four traits (Table 5). The variability of these estimates was uniformly small with 95th percentile interval widths exceeding no more than 0.11 .

\section{Discussion}

The calculation of BLUP values for panelists allowed for the adjustment of values at the individual apple level before

Table 4. Percentiles of time-averaged parental best linear unbiased prediction (BLUP) values and principal component (PC) scores relative to five apple families. ${ }^{\mathrm{z}}$

\begin{tabular}{lccc}
\hline & 'Honeycrisp' & MN-1702 & MN-1764 \\
\hline Crispness & 55 & 61 & 11 \\
Firmness & 31 & 68 & 5 \\
Juiciness & 95 & 55 & 70 \\
Total work to fracture & 15 & 73 & 4 \\
PC1 & 53 & 30 & 90 \\
PC2 & 1 & 60 & 2 \\
PC3 & 73 & 53 & 76 \\
PC4 & 52 & 39 & 52
\end{tabular}

${ }^{\text {zParental PC scores calculated using five-family derived loadings and }}$ scaling variables.
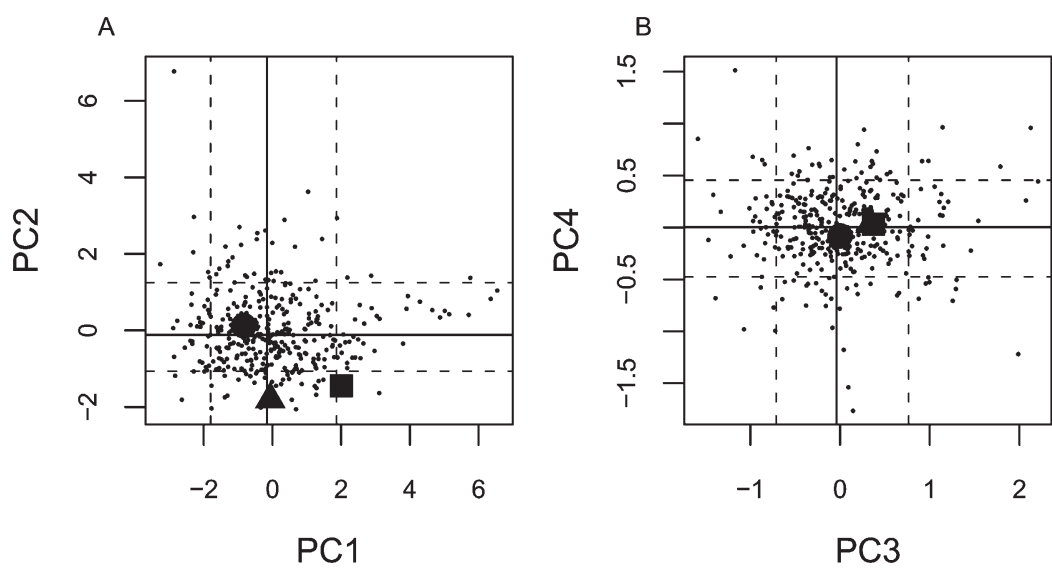

Fig. 2. Principal component (PC) scores derived from texture trait best linear unbiased prediction (BLUP) values of all five apple families averaged across years. Dashed lines indicate 10th and 90th percentiles, whereas solid lines indicate medians. Parental PC scores calculated using five familyderived PC loadings. Triangles indicate 'Honeycrisp', circles indicate MN-1702, and squares indicate MN-1764. (A) First two principal components, accounting for $44 \%$ and $28 \%$ of all variability, respectively. (B) Last two principal components, accounting for $17 \%$ and $11 \%$ of all variability, respectively. 'Honeycrisp' and MN-1764 symbols are superimposed in B. averaging within each genotype. The statistical significance of the panelist-by-year interaction terms seems to be driven by a small number of panelists with the majority of panelists exhibiting similar interannual variability (data not shown). This variability justifies the removal of panelist-by-year terms as part of the calculation of the panelist-adjusted predicted values. The implementation of training of the panelists could reduce some of this interannual variability in future studies. The inclusion of standards or controls could reduce further variability within panelists; for "at harvest" studies, which can initiate before the local harvest of commercial fruit, the acquisition of a large quantity of stored fruit at the onset of the study could provide standards for panelists, further reducing variability within panelists' assessments.

Including multiple fruit per genotype in panelists' samples introduced insignificant variability relative to samples from multiple genotypes, suggesting that effective trait characterization may be possible with even fewer fruit. This conclusion is consistent with the statement from Alspach and Oraguzie (2002) that a sample of only two fruit per tree was adequate for their own sensory evaluations. This observation suggests that large-scale population characterizations can occur when trees and their yields are small, albeit with the recognition that fruit quality may not be fully representative of potential quality under commercial conditions.

The inclusion of families containing reciprocal crosses allowed for the investigation of maternal or paternal effects. Direction of cross within family was not a statistically significant predictor for firmness, juiciness, or total work to fracture. Crispness, in contrast, demonstrated consistent directional effects over time as indicated by the statistical significance of the direction of cross within family and the lack of significant interaction between direction of cross and year. Further examination yields different parental effects depending on the family. When crossed with MN-1702, crispness was greater when 'Honeycrisp' was the paternal parent than for the reciprocal cross. The reverse pattern was observed when 'Honeycrisp' was crossed with MN-1764 and 'Jonafree'. Acknowledging unbalanced data and modest sample sizes, these differences are large enough to warrant further consideration as to whether the mechanisms are extranuclear or epigenetic in nature. The contradictory patterns among the different families suggest, however, that in the absence of further understanding of the process, apple breeders should consider making reciprocal crosses in pursuit of maximum variability from a single pair of parents.

Collection of the sensory data necessitated the removal of a quarter of each fruit, potentially initiating wound-induced texture changes (Lougheed and Franklin, 1974). As a result, within-fruit comparisons of sensory traits with instrumental traits were restricted to non-intact fruit. The time lag between sensory evaluations and the instrumental assay may have provided an opportunity for texture changes to initiate and accumulate. The inclusion of intact fruit from the same genotypes in 2005 and 2006 permitted an assessment of the relationships between the sensory traits and the instrumental trait but at 
Table 5. Broad-sense heritability estimates, represented as mean bootstrapped correlations, and corresponding 95th percentile intervals for apple fruit sensory traits and instrumental measures of total work to fracture. ${ }^{z}$

\begin{tabular}{lcc}
\hline & Mean correlation & 95th Percentile interval \\
\hline Crispness & 0.76 & $0.73-0.80$ \\
Firmness & 0.81 & $0.78-0.85$ \\
Juiciness & 0.72 & $0.66-0.77$ \\
Total work to fracture & 0.75 & $0.72-0.79$ \\
\hline
\end{tabular}

${ }^{\mathrm{z}}$ Crispness, firmness, and juiciness estimates were calculated using back-transformed data (see text).

the genotype level rather than at the within-fruit level. Despite the high correlation between intact and non-intact values, all three sensory traits were more highly correlated to the nonintact fruit total work to fracture values, derived from the same fruit, than to the intact fruit values (data not shown), derived from separate fruit. This suggests that the introduction of potential wound-induced texture changes affects total work to fracture less than the introduction of new, intact fruit into the study and supports the retention of non-intact fruit in cold storage for 1 week or more as opposed to the use of separate fruit for separate measures.

Mann et al. (2005) reported total work to fracture as a statistically significant predictor of sensory crispness $\left(r^{2}=0.67\right)$ and force at fracture as a predictor for firmness $\left(r^{2}=0.43\right)$. Corresponding values relating this study's instrumental measure, total work to fracture, with two of the sensory traits, panelistadjusted crispness and firmness, were comparable $\left[r=0.47\left(r^{2}=\right.\right.$ $0.69)$ and $r=0.62\left(r^{2}=0.38\right)$, respectively; Table 2]. These similar results likely reflect common growing conditions and some common ancestry, because Mann et al. (2005) primarily evaluated a small number of advanced selections from the same breeding program.

The PCA illustrates the benefit of simultaneously phenotyping several traits. The notable texture of 'Honeycrisp' stands out once the collinearity of the four texture traits has been accounted for by the first principal component as a combination of high juiciness with low total work to fracture expressed by the second principal component. The first percentile ranking of 'Honeycrisp' with respect to this component suggests that replicating this trait in future genotypes is likely to be a very rare event using traditional breeding methods.

Narrow-sense heritability values for sensory texture traits in apple have ranged from 0.12 to 0.59 (Alspach and Oraguzie, 2002; King et al., 2000; Kouassi et al., 2009) across a wide array of scenarios and genetic breadth. The estimates of broad-sense heritability, $\mathrm{H}^{2}$, in the current study are consistently greater than previously published narrow-sense estimates. This is to be expected, because $\mathrm{H}^{2}$ is greater than or equal to $\mathrm{h}^{2}$; the latter term only incorporates additive and additive epistatic variances, whereas the former term also incorporates dominance and other epistatic variances. The inclusion in the study of multiple families known to segregate with respect to texture traits would be expected to increase genetic variability relative to phenotypic variability and lack of common ancestry among any of the parental genotypes would increase this effect. The implementation of multiple harvests would be expected to increase phenotypic variability, but also to increase the ability to partition genetic and environmental variability, increasing heritability estimates.
Although estimates of narrow-sense heritability are more informative for estimating the breeding value of an individual, the interpretation of this parameter's estimates are rendered unclear by highly unbalanced populations. Nonetheless, broadsense heritability is a very useful parameter for clonally propagated crops, individual per se performance being the basis of selecting among individuals (e.g., Poehlman and Sleper, 1995). Long generation times restrict the number of recombination opportunities, and thus linkages between genes are likely to be retained in a breeding program relative to annual crops. The asexual propagation of most tree fruit crops further ensures that desirable linkages are retained within desirable phenotypes so long as a substantial fraction of the phenotypic variation is genetically determined. In other words, total genetic variance contributing to high broad-sense heritability, regardless of the relative proportions of its constituent variance components, can be maintained through asexual propagation, resulting in high levels of crop uniformity.

Mixed effects linear models incorporating ReML have been used to analyze complex population structures and unbalanced experimental designs (e.g., Alspach and Oraguzie, 2002; Durel et al., 1998). The choice of parental genotypes with no known common ancestors likely limits the use of recent pedigreebased methods (e.g., Bink et al., 2008; Kouassi et al., 2009). Additionally, the simplicity of the population structure is likely to reduce the influence of correlations inherent among related plants; the unbalanced nature of the data set is more likely to complicate the interpretation of the heritability values. Direct application of heritability estimates to a selection protocol is unwarranted (Piepho and Möhring, 2007). Nonetheless, this analysis suggests that continued selection for enhanced fruit texture traits is likely to be productive.

\section{Conclusions}

The rapid development of new apple cultivars requires the efficient, early characterization of traits of interest within the breeding population as well as heritability estimates for the same traits to provide guidance in the design of subsequent crosses within a breeding program. The large space and time requirements for breeding tree crops results in many genetic studies being based on active breeding programs as opposed to populations especially designed to address genetic questions. 'Honeycrisp' is represented in many different crosses, including several reciprocal crosses. The other five parents, in contrast, are of lesser interest despite their potential for contribution of desirable genetic traits into the 'Honeycrisp' background. As a result, these other parents are rarely crossed with each other and the existing structure of this breeding program does not contain diallel-type population designs as are frequently developed for genetic studies. Nonetheless, breeding populations can yield valuable information, particularly with respect to phenotypic characterization.

Protocols that aid in the collection of phenotypic data can have substantial financial and labor impacts for a breeding and research program. This study combined sensory evaluation panels, typically restricted to advanced selections in apple breeding programs (e.g., Hampson et al., 2000), with incomplete block design and mixed-effect modeling to characterize several fruit texture traits in unselected seedling populations. The randomized incomplete block design accommodated the evaluation of many genotypes by moderately sized sensory 
evaluation panels and small quantities of fruit per genotype. The inclusion of replicated genotypes within individual panelists' samples confirmed that between-genotype variability far exceeded within-genotype variability. The repeated participation of panelists in successive panels permitted the evaluation of panelist effects and subsequent adjustment of phenotypic values. The inclusion of parental genotypes in the analyses provided a basis by which to assess the predicted values. Taken together, these methods have enabled the evaluation of a large number of genotypes from an active breeding population at a young age. This study suggests that the desirable texture traits of the cultivar Honeycrisp reflect an unusual combination of multiple component traits, each of which express high levels of broad-sense heritability within this breeding population. These results suggest that continued breeding using 'Honeycrisp'-derived germplasm holds promise for further improvement of fruit texture traits.

\section{Literature Cited}

Allan-Wojtas, P., K.A. Sanford, K.B. McRae, and S. Carbyn. 2003. An integrated microstructural and sensory approach to describe apple texture. J. Amer. Soc. Hort. Sci. 128:381-390.

Alspach, P.A. and N.C. Oraguzie. 2002. Estimation of genetic parameters of apple (Malus domestica) fruit quality from open-pollinated families. N. Z. J. Crop Hort. Sci. 30:219-228.

Bates, D. 2007. lme4: Linear mixed-effects models using S4 classes. R package version 0.99875-9. 3 Mar. 2011. <http://cran.r-project.org/ web/packages/lme4/index.html>.

Bink, M.C.A.M., M.P. Boer, C.J.F. ter Braak, J. Jansen, R.E. Voorrips, and W.E. van de Weg. 2008. Bayesian analysis of complex traits in pedigreed plant populations. Euphytica 161:85-96.

Blanpied, G.D. and K.J. Silsby. 1992. Predicting harvest date windows for apples. Cornell Coop. Ext. Info. Bul. 221:1-12.

Chauvin, M.A., C.F. Ross, M. Pitts, E. Kaupferman, and B. Swanson. 2010. Relationship between instrumental and sensory determination of apple and pear texture. J. Food Qual. 33:181-198.

Daillant-Spinnler, B., H.J.H. MacFie, P.K. Beyts, and D. Hedderley. 1996. Relationships between perceived sensory properties and major preference direction of 12 varieties of apples from the southern hemisphere. Food Qual. Prefer. 7:113-126.

Durel, C.E., F. Laurens, A. Fouillet, and Y. Lespinasse. 1998. Utilization of pedigree information to estimate genetic parameters from large unbalanced data sets in apple. Theor. Appl. Genet. 96:1077-1085.

Food and Agricultural Organization of the United Nations. 2010. FAOSTAT. 8 Apr. 2011. <http://faostat.fao.org>.

Green, B.G., G.S. Shaffer, and M.M. Gilmore. 1993. Derivation and evaluation of a semantic scale of oral sensation magnitude with apparent ratio properties. Chem. Senses 18:683-702.

Hampson, C.R., H.A. Quamme, J.W. Hall, R.A. MacDonald, M.C. King, and M.A. Cliff. 2000. Sensory evaluation as a selection tool in apple breeding. Euphytica 111:79-90.

Harker, F.R., K. Lau, and F.A. Gunson. 2003. Juiciness of fresh fruit: A time-intensity study. Postharvest Biol. Technol. 29:55-60.
Harker, F.R., J. Maindonald, S.H. Murray, F.A. Gunson, I.C. Hallett, and S.B. Walker. 2002. Sensory interpretation of instrumental measurements 1: Texture of apple fruit. Postharvest Biol. Technol. 24:225-239.

Janick, J., J.N. Cummins, S.K. Brown, and M. Hemmat. 1996. Apples, p. 1-77. In: Janick, J. and J.N. Moore (eds.). Fruit breeding, Volume I: Tree and tropical fruits. Wiley, New York, NY.

John, J.A. and E.R. Williams. 1995. Cyclic and computer generated designs. 2nd Ed. Chapman and Hall, London, UK.

Karlsen, A.M., K. Aaby, H. Sievertsen, P. Baardseth, and M.R. Ellekjær. 1999. Instrumental and sensory analysis of fresh Norwegian and imported apples. Food Qual. Prefer. 10:305-314.

King, G.J., C. Maliepaard, J.R. Lynn, F.H. Alston, C.E. Durel, K.M. Evans, B. Griffon, F. Laurens, A.G. Manganaris, E. Schrevens, S. Tartarini, and J. Verhaegh. 2000. Quantitative genetic analysis and comparison of physical and sensory descriptors relating to fruit flesh firmness in apple (Malus pumila Mill.). Theor. Appl. Genet. 100:1074-1084.

Kouassi, A.B., C.-E. Durel, F. Costa, S. Tartarini, E. van de Weg, K. Evans, F. Fernandez-Fernandez, C. Govan, A. Boudichevskaja, F. Dunemann, A. Antofie, M. Lateur, M. Stankiewicz-Kosyl, A. Soska, K. Tomala, M. Lewandowski, K. Rutkovski, E. Zurawicz, W. Guerra, and F. Laurens. 2009. Estimation of genetic parameters and prediction of breeding values for apple fruit-quality traits using pedigreed plant material in Europe. Tree Genet. Genomes 5:659672.

Lougheed, E.C. and E.W. Franklin. 1974. Ethylene production increased by bruising of apples. HortScience 9:192-193.

Mann, H., D. Bedford, J. Luby, Z. Vickers, and C. Tong. 2005. Relationship of instrumental and sensory texture measurements of fresh and stored apples to cell number and size. HortScience 40:18151820.

Oraguzie, N.C., M.E. Hofstee, L.R. Brewer, and C. Howard. 2001. Estimation of genetic parameters in a recurrent selection program in apple. Euphytica 118:29-37.

Péneau, S., E. Hoehn, H.-R. Roth, F. Escher, and J. Nuessli. 2006. Importance and consumer perception of freshness of apples. Food Qual. Prefer. 17:9-19.

Piepho, H.P. and J. Möhring. 2007. Computing heritability and selection response from unbalanced plant breeding trials. Genetics 177:1881-1888.

Piepho, H.P., J. Möhring, A.E. Melchinger, and A. Büchse. 2008. BLUP for phenotypic selection in plant breeding and variety testing. Euphytica 161:209-228.

Piepho, H.P., E.R. Williams, and M. Fleck. 2006. A note on the analysis of designed experiments with complex treatment structure. HortScience 41:446-452.

Pinheiro, J.C. and D.M. Bates. 2002. Mixed effects models in S and S-Plus. Springer, New York, NY.

Poehlman, J.M. and D.A. Sleper. 1995. Breeding field crops. Iowa State Univ. Press, Ames, IA.

R Development Core Team. 2007. R: A language and environment for statistical computing. 3 Apr. 2011. <http://www.R-project.org>.

Whitaker, D., E.R. Williams, and J.A. John. 2004, CycDesigN: A package for the computer generation of experimental designs. Version 2.1. CSIRO Forestry and Forest Products, Canberra, Australia. 